\title{
Hiperplasia Fibrosa Inflamatoria: reporte de un caso
}

\author{
Inflammatory Fibrous Hyperplasia: case report
}

Casian Romero A ${ }^{1}$, Trejo Quiroz P², De León Torres $C^{3}$, Carmona Ruiz D4

\section{RESUMEN}

Introducción: La Hiperplasia Fibrosa Inflamatoria es una alteración caracterizada por un agrandamiento del tejido conectivo; se considera una lesión reactiva ya que se presenta como respuesta a una irritante crónico local; es una lesión común en pacientes adolescentes y de edad adulta debido a que su etiología principal se asocia a maloclusión, al uso de aparatología protésica u ortodóntica mal ajustada, así como a la presencia de biofilm. Sin embargo, la población infantil, principalmente en dentición mixta, no está exenta de presentar esta alteración, aunque en menor proporción. Objetivo: Presentar el caso clínico de un paciente en dentición mixta que presenta una Hiperplasia Fibrosa Inflamatoria con el fin de presentar las características clínicas e histopatológicas de la lesión, enfatizando la importancia de un temprano y correcto diagnóstico. Presentación del Caso: Paciente masculino de 8 años de edad que se presenta a la clínica por presentar lesiones cariosas y malposición dental. A la exploración se observa un agrandamiento gingival localizado, en el órgano dentario 41. Se decide tomar una biopsia de la lesión para establecer el diagnóstico. Conclusión: Debido a que existen varias lesiones con características clínicas muy similares a las que presentaba el paciente, es necesario conocer los diagnósticos diferenciales, así como realizar el diagnóstico temprano de la lesión para poder llevar a cabo un tratamiento eficaz y adecuado.

Rev. Clin. Periodoncia Implantol. Rehabil. Oral Vol. 4(2); 74-79, 2011.

Palabras clave: Hiperplasia, biofilm, maloclusión dental, agrandamiento gingival.

\section{ABSTRACT}

Introduction: The Inflammatory Fibrous Hyperplasia (IFH) is a disorder consisting in a connective tissue overgrowth; it is considered a reactive lesion because it appears in response to a chronic and local irritant; this is a very common oral lesion in adolescents and adults, its etiology is associated with dental malocclusion, use of over-extended prosthetic and orthodontic appliances, and with the presence of biofilm (plaque). Nevertheless, child population, mainly patients in mixed dentition, are not exempt from presenting this lesion, in a lower proportion, though. Objective: The aim of this case report is to present the clinical case of a patient in mixed dentition, who displays an Inflammatory Fibrous Hyperplasia, with the purpose of showing the clinical and histopathological features of this lesion, emphasizing the importance of an early and correct diagnosis. Case Report: A 8 year-old male patient, was referred to the Pediatric Dentistry Clinic of the Division of Post-graduate studies and Research, of the School of Dentistry, UNAM, for showing decay lesions and dental malposition. Clinical examination revealed a localized gingival over-growth associated to the teeth 41 . It was decided to take a biopsy of the lesion to establish the final diagnosis. Conclusion: Because there are numerous lesions with pretty similar clinical features to the ones the patient presented, it is imperative and essential to know the differential diagnosis of this lesion, and to achieve an early diagnosis to accomplish an effective and appropriate treatment.

Rev. Clin. Periodoncia Implantol. Rehabil. Oral Vol. 4(2); 74-79, 2011.

Key words: Verrucous carcinoma, case report.

\section{INTRODUCCIÓN}

El término de Hiperplasia se refiere a un aumento de volumen de un tejido producido por el incremento en el número de sus células; en general, corresponden a un grupo de lesiones producidas como una respuesta exagerada de la mucosa bucal frente a irritantes crónicos de baja intensidad, motivo por el cual se le considera una lesión reactiva ${ }^{(1)}$.

Las Hiperplasias se clasifican en base al tipo de tejido que las conforman, pudiendo ser: con predominio de tejido de granulación (como es en el caso de un Granuloma Piógeno), con predominio de tejido fibroso (como las Hiperplasias Fibrosas Inflamatorias) o clasificadas como otras lesiones Hiperplásicas (como la Hiperplasia Gingival inducida por Fármacos) $)^{(2)}$.

La Hiperplasia Fibrosa Inflamatoria (HFI) es un agrandamiento reactivo, localizado y de tipo inflamatorio del tejido conectivo, más frecuente en adolescentes y adultos y relativamente común en niños (menos del 5\%). Lesión de crecimiento lento y generalmente asintomática, considerada como un aumento celular proliferativo noneoplásico en respuesta a la acción de agentes físicos constantes ${ }^{(3)}$.

Su etiología es variada y se asocia a la presencia de factores irritativos como la presencia de biofilm, cálculo, aparatología ortodóntica o protésica mal ajustada o sobreextendida, malposición dental y por desequilibrios hormonales ${ }^{(4)}$. En las primeras etapas el irritante crónico estimula la formación de tejido de granulación; más adelante, el tejido comienza a sufrir un proceso de fibrosis. La presencia de factores irritativos en la mucosa desencadena un proceso inflamatorio crónico que culmina con la formación de tejido fibroso hiperplásico, asintomático, en las zonas de agresión, conocida como Hiperplasia Fibrosa Inflamatoria $(\mathrm{HIF})^{(5)}$.

Las lesiones de naturaleza inflamatoria constituyen aproximadamente el $66 \%$ de todas las lesiones de la cavidad oral, siendo la mayoría de estas lesiones atribuidas a la mala higiene y al uso de aparatología fija o removible(2).

La mayoría de los casos han sido reportados entre la cuarta y sexta década de vida, determinándose una relación directa entre la frecuencia de esta lesión con el aumento del período del uso de las prótesis; una minoría (menos del $5 \%$ ) de los casos se presentan en niños, principalmente en aquellos que se encuentran en dentición mixta. En adultos está principalmente asociada a pacientes que utilizan prótesis bucales mal adaptadas; en niños y adolescentes, asociada a presencia de biofilm, malposición dental y aparatología fija o removible ${ }^{(6)}$; sin embargo, existe poca literatura sobre la prevalencia de éstas lesiones en la población mexicana.

La HFI posee una frecuencia alta y constituye la lesión oral más prevalente en adultos.

La HFI es una lesión que no presenta una predisposición

1. Residente. $2^{\circ}$ año de la Especialidad de Odontopediatría, DEPel, Facultad de Odontología, UNAM. México.

2. Profesora. Especialidad de Odontopediatría, DEPel, Facultad de Odontología, UNAM. México.

3. Profesora. Odontopediatría, División de Estudios Profesionales, Facultad de Odontología, UNAM. México.

4. Profesora. Odontología Preventiva, División de Estudios Profesionales, Facultad de Odontología, UNAM. México. 
por sexo, sin embargo, se cree que el sexo femenino es más proclive a presentar esta lesión, en una proporción de 5:1, lo cual puede deberse al hecho de que las mujeres utilizan más frecuentemente prótesis o aparatos ortodónticos probablemente por razones estéticas ${ }^{(7)}$.

Puede manifestarse en cualquier localización intraoral, pero la zona más frecuente de aparición es la región gingival, seguida por la mucosa vestibular, lengua, labios y el paladar. Ha sido relatada con mayor frecuencia en la región del surco vestibular y, en menor proporción, en reborde alveolar lingual de mandíbula y en la región palatina. Afecta principalmente a la zona anterior de mandíbula y maxilar, aunque también puede afectar con menor frecuencia la zona posterior, no existiendo predilección por maxila o mandíbula ${ }^{(8)}$. La HFI se puede clasificar por su localización en:

Marginal. Circunscrito en la encía marginal.

Papilar. Se limita solamente a la paila interdental.

Difusa. Afecta a la encía marginal insertada y papilar.

La lesión se presenta como un agrandamiento gingival del tejido conectivo, de crecimiento lento y generalmente asintomático, de color de rosado pálido semejante a la mucosa adyacente; su diámetro varía de menos de $1 \mathrm{~cm}$ a lesiones grandes que pueden afectar la totalidad del vestíbulo. Los casos que presentan sintomatología están asociados a ulceración concomitante de la lesión; se muestra eritematoso si está ulcerado, semejando un Granuloma Piógeno. La consistencia de la lesión es variada de firme a flácida; puede presentarse con una superficie lisa, con base sésil y ocasionalmente pediculada; sangra fácilmente a la palpación o al cepillado(9).

Histológicamente se caracteriza por una sobreproducción de tejido conjuntivo fibroso, rico en fibras de colágeno y fibroblastos, delimitado por epitelio escamoso superficial; existe presencia de infiltrado inflamatorio crónico (linfocitos y células plasmáticas, principalmente y ocasionalmente leucocitos polimorfonucleares pueden estar presentes) y pocos vasos sanguíneos. El aspecto del tejido conjuntivo varía de acuerdo con el estado de desarrollo de la lesión: de tejido de granulación inmaduro en lesiones jóvenes a tejido conjuntivo denso y fibroso en lesiones más antiguas. En muchos casos una misma lesión puede presentar diferentes aspectos microscópicos ${ }^{(10)}$.

El diagnóstico de HFI generalmente es clínico establecido por la relación entre la causa y consecuencia de la lesión; y por medio de un estudio histopatológico (biopsia excisional). Ocasionalmente se requiere de exámenes radiográficos para descartar el comprometimiento óseo ${ }^{(11)}$.

Sus principales diagnósticos diferenciales son:

\section{Granuloma Piógeno.}

Granuloma del Embarazo.

Fibroma Traumático.

Hiperplasia Papilar Inflamatoria.

Granuloma Periférico de Células Gigantes.

Fibromatosis Gingival.

Hiperplasia Gingival Inducida por Fármacos ${ }^{(12)}$.

El tratamiento va de acuerdo con la severidad de la lesión y la cantidad del tejido afectado; lesiones pequeñas pueden ser tratadas de manera conservadora, con remoción del agente, mientras que lesiones extensas y de larga evolución necesitarán de excisión quirúrgica con posterior biopsia. En cualquier caso es inevitable la erradicación del factor irritativo ${ }^{(13)}$.

El pronóstico de la HFI es bueno y los índices de recidivas son bajos siempre y cuando el factor irritativo sea eliminado ${ }^{(14)}$.

\section{OBJETIVO}

El objetivo de este artículo es presentar el caso clínico de un paciente en dentición mixta con el fin de establecer las características clínicas e histopatológicas de la Hiperplasia Fibrosa Inflamatoria, una lesión no del todo común en la población infantil, así como enfatizar la importancia de un buen diagnóstico de las lesiones en la cavidad oral no solo en niños, sino en adultos también.

\section{REPORTE DEL CASO CLÍNICO}

Se presenta en la Clínica de Odontopediatría de la División de Estudios de Posgrado e Investigación de la Facultad de Odontología de la UNAM. Un paciente masculino de 8 años de edad, el cual acude para tratamiento de caries dental. Dentro de sus antecedentes heredofamiliares la madre refiere antecedentes de Hipertensión y Diabetes Mellitus por parte de los abuelos paternos y maternos. El paciente refiere una higiene bucal deficiente, ya que lo realiza únicamente una vez al día. En los antecedentes personales patológicos se refiere que el paciente padeció Varicela, como única enfermedad de la infancia, a los 6 años de edad.

A la exploración clínica se encontraron múltiples lesiones cariosas de $2^{\circ}$ grado, que requerían de tratamiento de operatoria así como restauraciones previas (Figura 1).

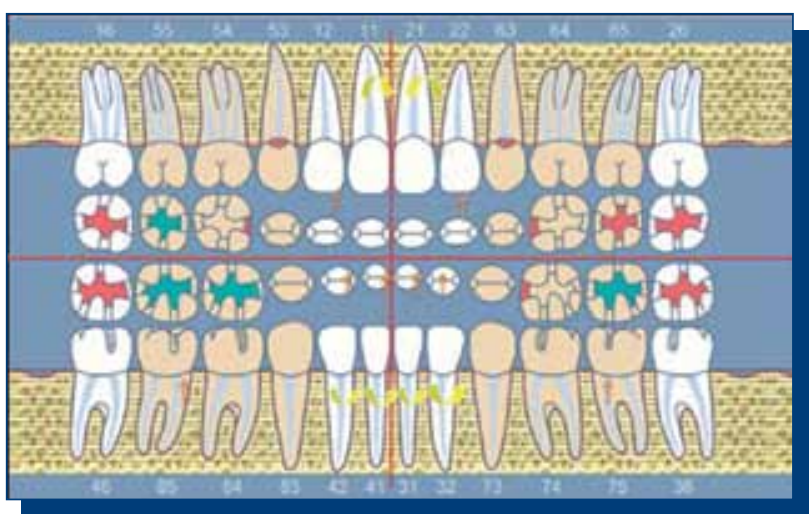

Figura 1. Odontograma inicial del paciente. Con rojo se observan caries y en verde las restauraciones previas al momento de realizar la Historia Clínica.

También, se observó sobremordida vertical aumentada y malposición dental en varios órganos dentarios (Figuras 2, 3, 4 y 5). De igual forma se encontró un agrandamiento gingival, eritematoso, que sangraba fácilmente a la palpación y al cepillado, en el margen gingival del órgano dentario 41 . El diente presentaba malposición dental (giroversión hacia Mesial) y presencia de abundante biofilm (Figuras 6, 7 y 8). Radiográficamente, no se observaron hallazgos significativos (Figuras 9 y 10).

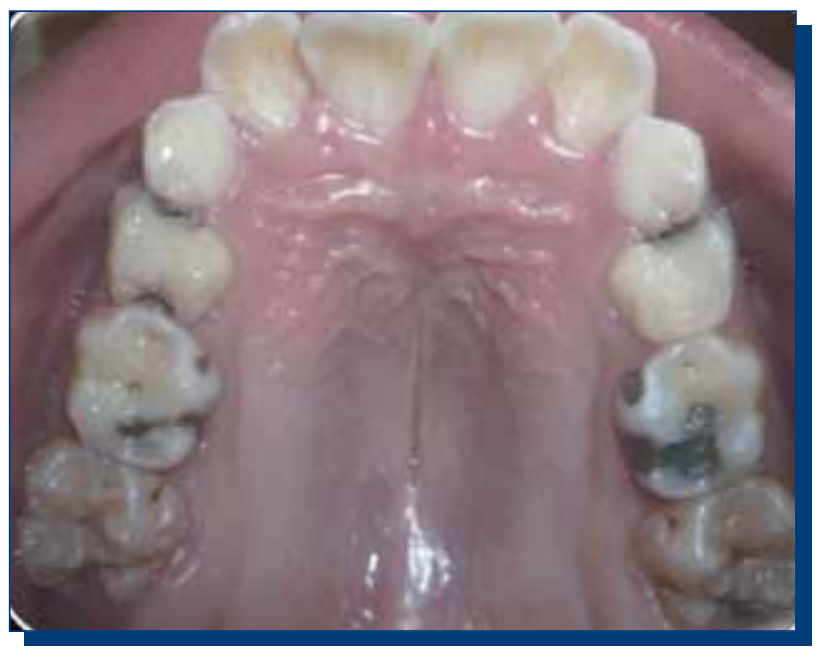

Figura 2. Vista oclusal superior. 


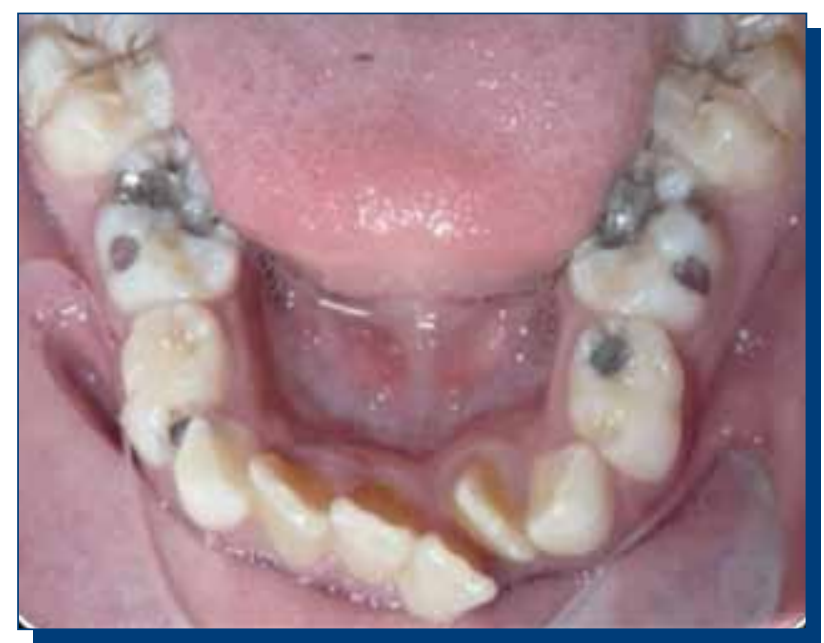

Figura 3. Vista oclusal inferior, donde se observa el marcado apiñamiento en la zona anterior inferior.

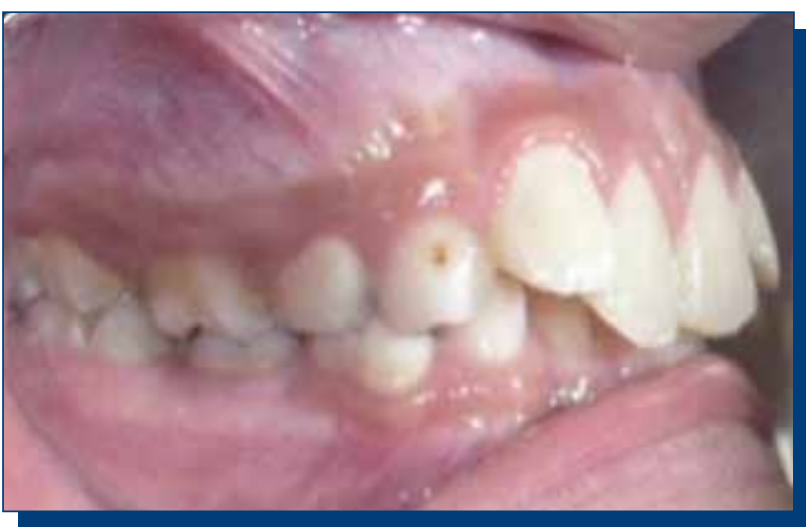

Figura 4. Vista lateral derecha.

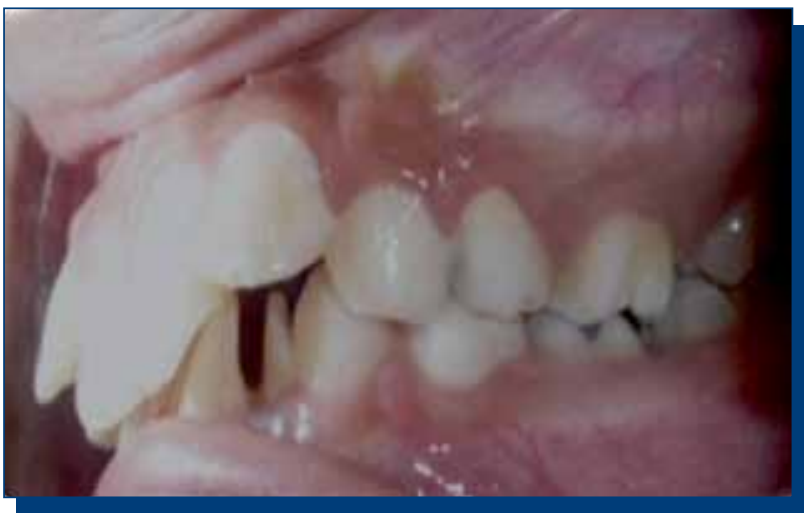

Figura 5. Vista lateral izquierda.

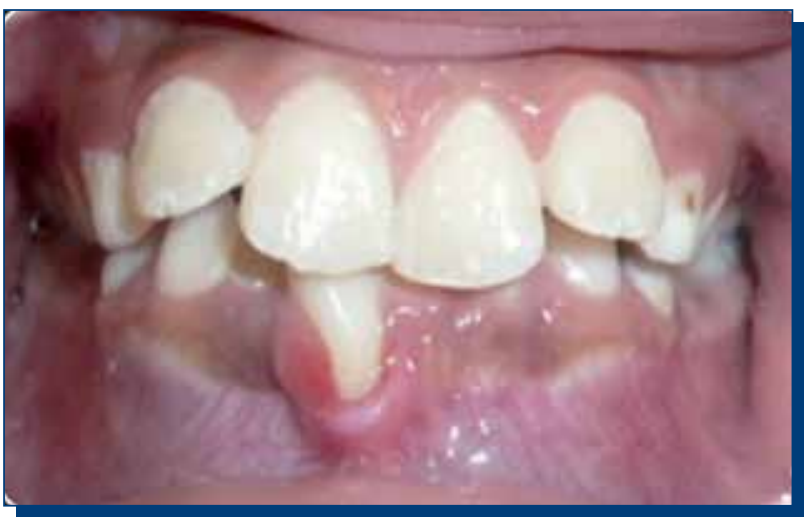

Figura 6. Vista anterior en donde se observa la marcada sobremordida vertical y el agrandamiento gingival del O.D. 41.
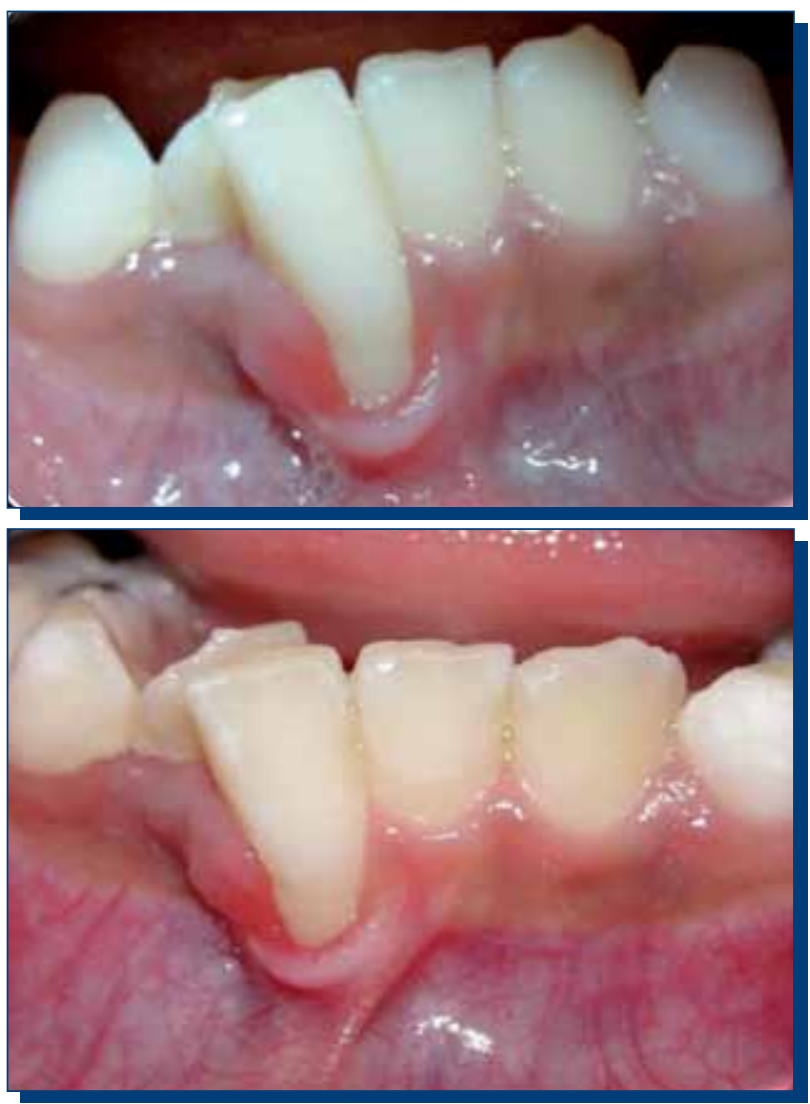

Figuras 7 y 8 . Aspecto clínico inicial de la lesión.

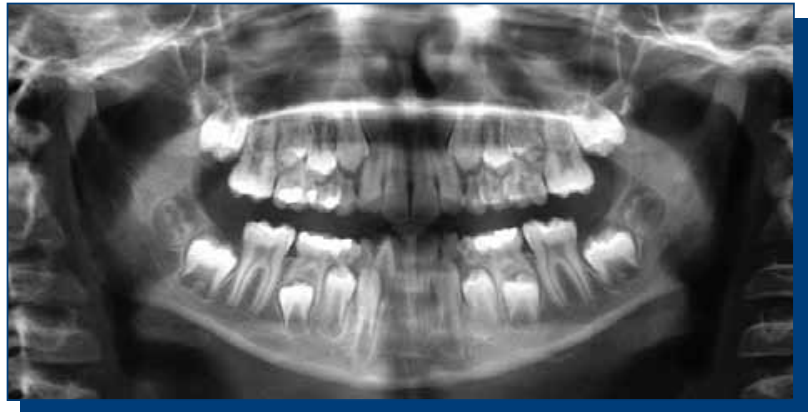

Figura 9. Ortopantomografía.

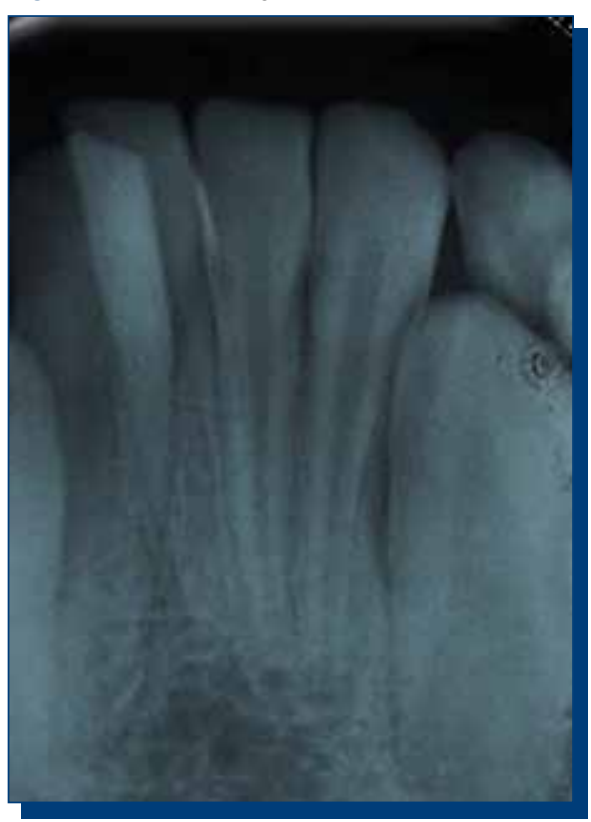

Figura 10. Aspecto radiográfico de la lesión. 
Como primera fase de tratamiento se realizaron controles personales de placa, profilaxis, aplicación tópica de flúor y se dió técnica de cepillado al paciente y a la madre.

En esa misma etapa se llevó a cabo la rehabilitación de las lesiones cariosas, la cual consistió en tratamientos de operatoria dental y prevención (eliminación de caries y posterior colocación de resinas, ionómeros de vidrio y selladores de fosetas y fisuras) (Figuras 11 y 12). Como segunda etapa se propuso la valoración para realizar un tratamiento de ortopedia maxilar.

En cuanto a la lesión gingival del órgano dentario 41, debido a que no cedió al mejorar la higiene con el transcurso de los días, se realizó interconsulta con el departamento de periodoncia para valorar la lesión. Se decidió tomar una biopsia excisional con el fin de establecer el diagnóstico, bajo la sospecha de que la lesión fuese un Granuloma Piógeno (Figuras 13 y 14).

La biopsia realizada fue de tipo excisional; la muestra obtenida midió $10 \mathrm{~mm} \times 5 \mathrm{~mm}$ y fue enviada al Laboratorio de Patología Bucal de la División de Estudios de Posgrado e Investigación de la Facultad de Odontología, UNAM, para realizar el examen histopatológico (Figuras 15 y 16).
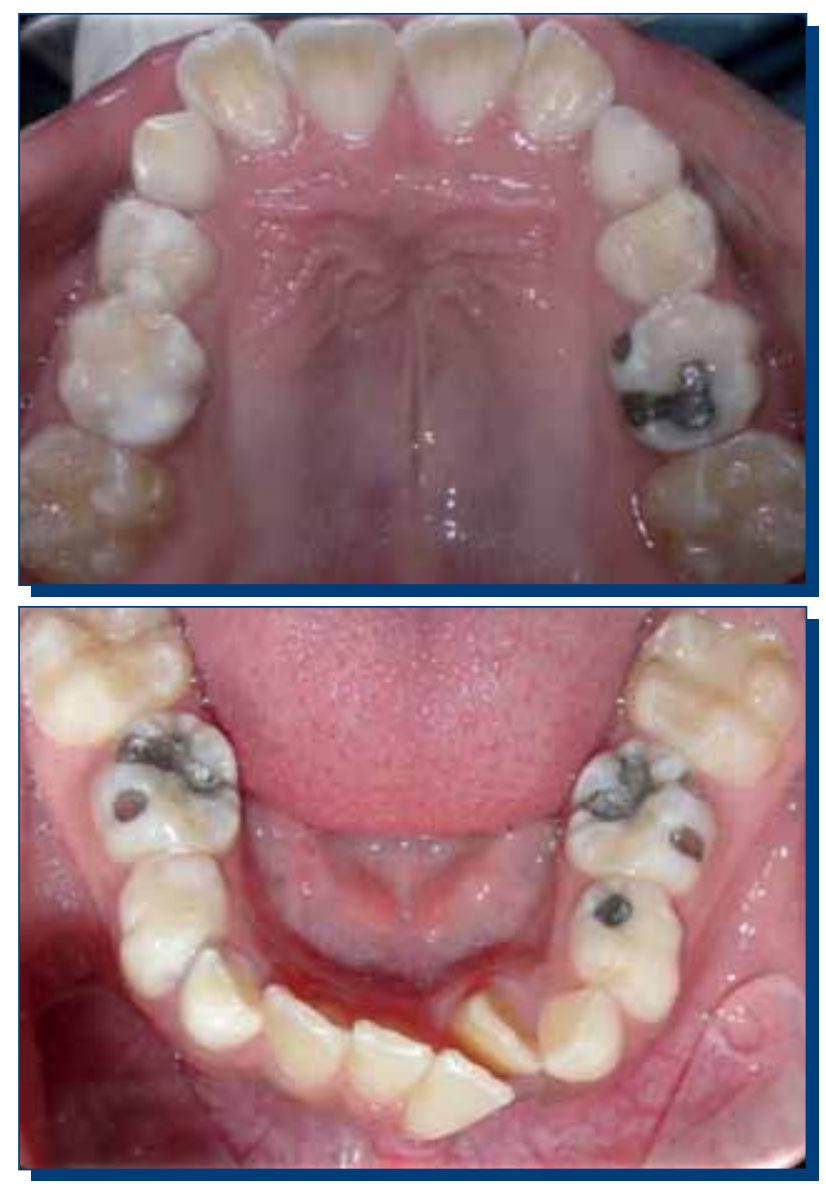

Figuras 11 y 12. Vistas oclusales tras la rehabilitación de operatoria.
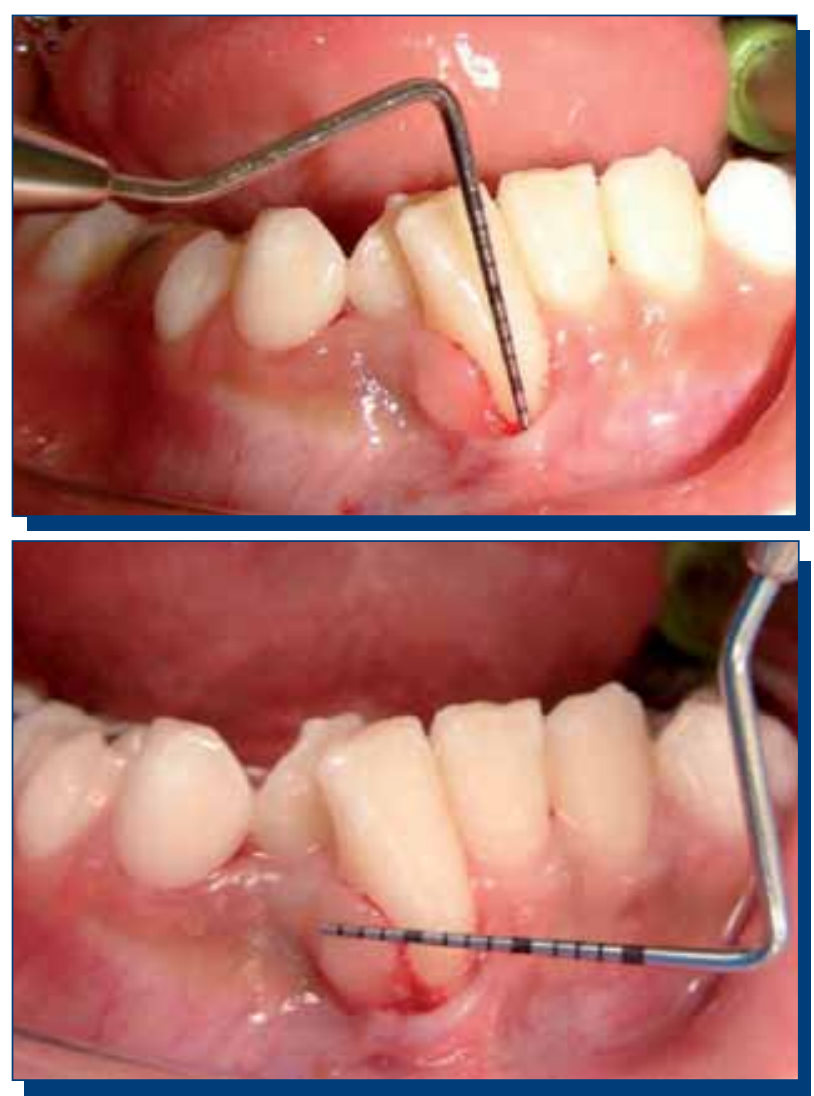

Figuras 13 y 14. Mediciones del agrandamiento previo a la toma de biopsia.

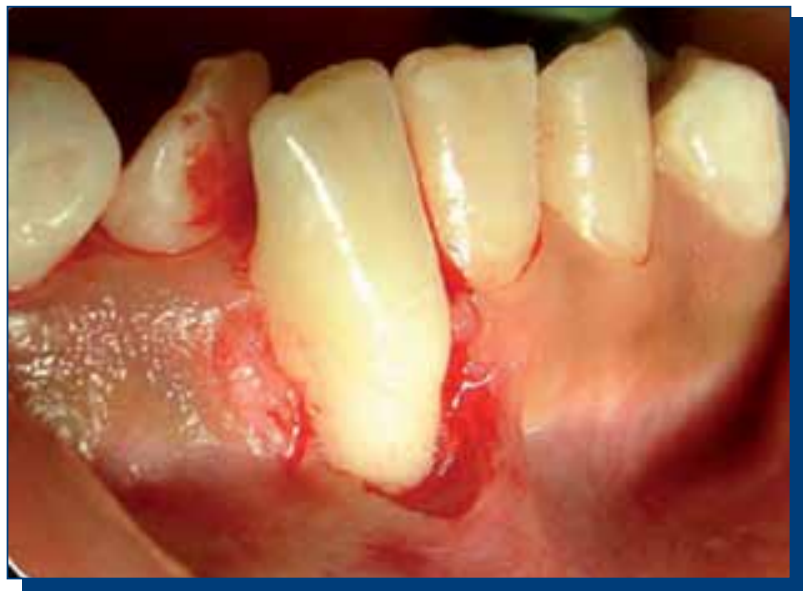

Figura 15. Aspecto del diente tras la toma de biopsia, donde se observa la recesión gingigval. 


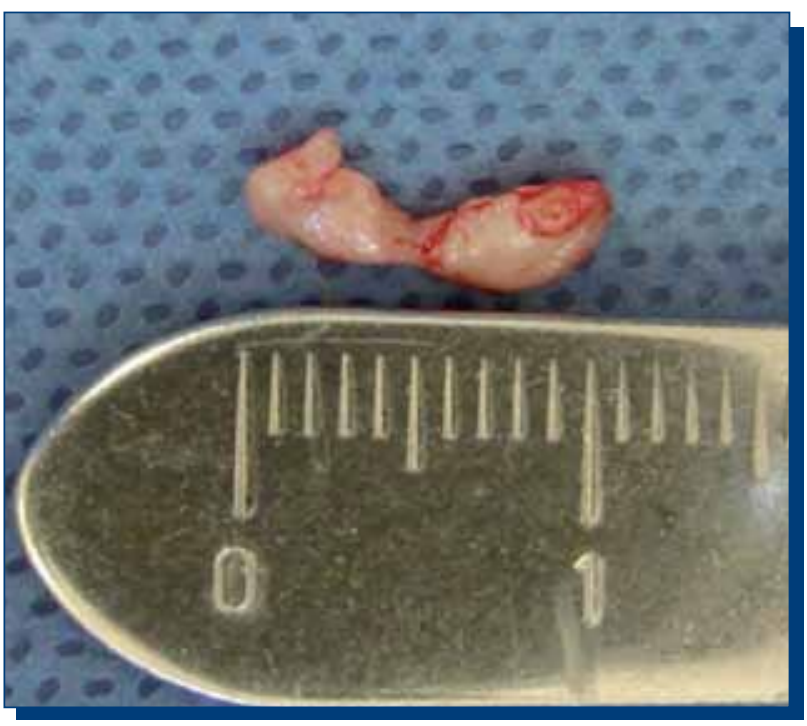

Figura 16. Muestra obtenida lista para su estudio histopatológico.

\section{RESULTADOS}

El estudio histopatológico mostró un alto contenido de tejido conjuntivo fibroso denso, bien vascularizado, Infiltrado inflamatorio crónico severo difuso, parcialmente cubierto por epitelio escamoso estratificado (Figuras 17, 18 y 19), lo que reveló el diagnóstico de Hiperplasia Gingival Inflamatoria, asociada a la presencia de malposición dental y biofilm en el diente.

Debido a la recesión gingival obtenida al realizar la biopsia (Figuras 20, 21, 22 y 23), se determinó que en la siguiente fase se enfatizará en la higiene oral del paciente y se dará inicio con la ortopedia dentomaxilar, para corregir el apiñamiento de la zona.

Como tercera fase y con ayuda del Departamento de Periodoncia de la DEPEI, UNAM, se realizará un Injerto Mucogingival en la zona de recesión una vez finalizado el tratamiento de ortopedia.

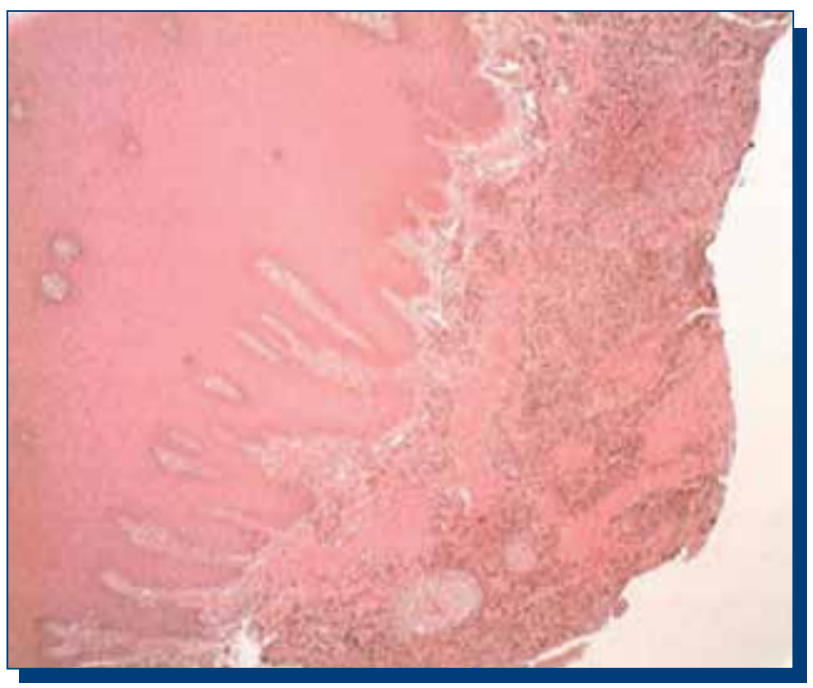

Figura 17. Se observa epitelio escamoso estratificado y tejido conjuntivo subyacente.

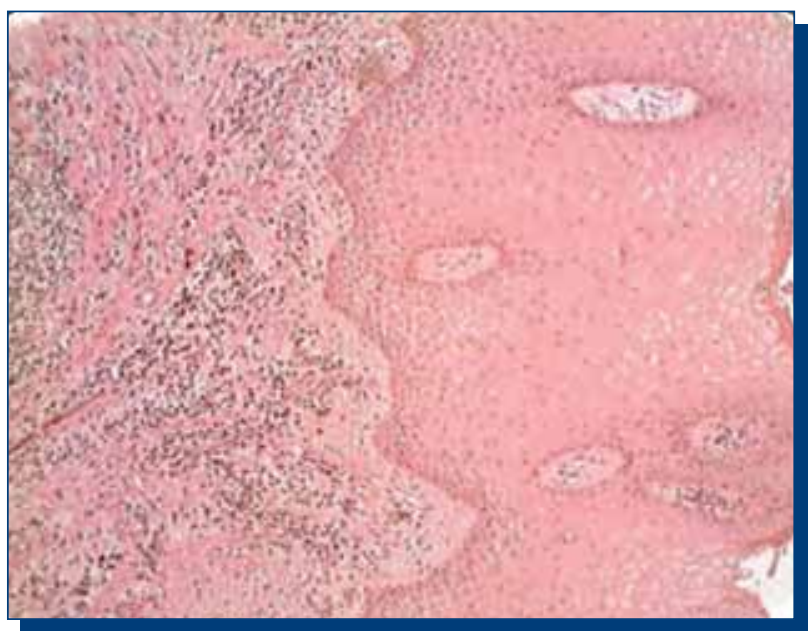

Figura 18. Se observa epitelio con acantosis y edema intracelular.

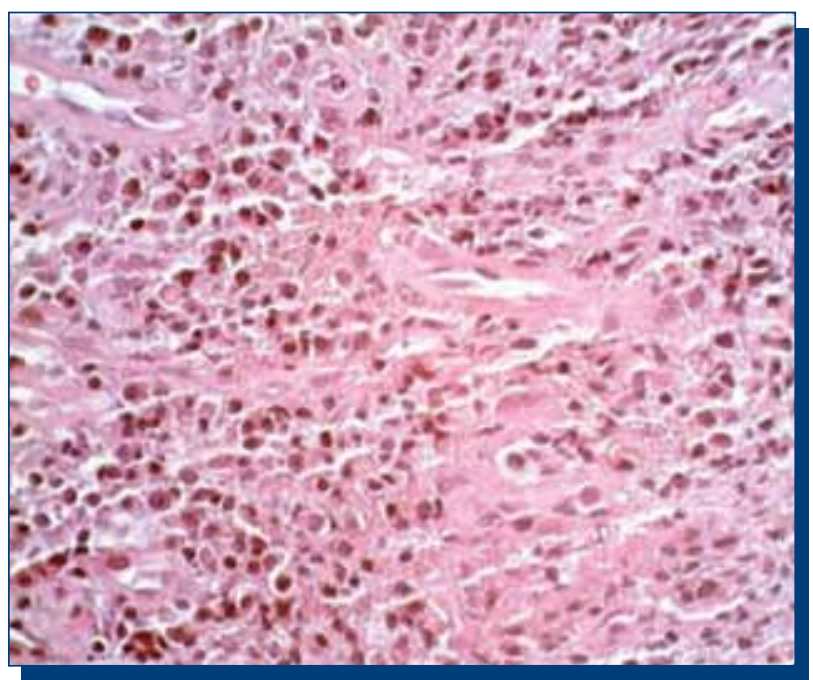

Figura 19. Se observa tejido conjuntivo fibroso denso e infiltrado inflamatorio crónico moderado difuso.

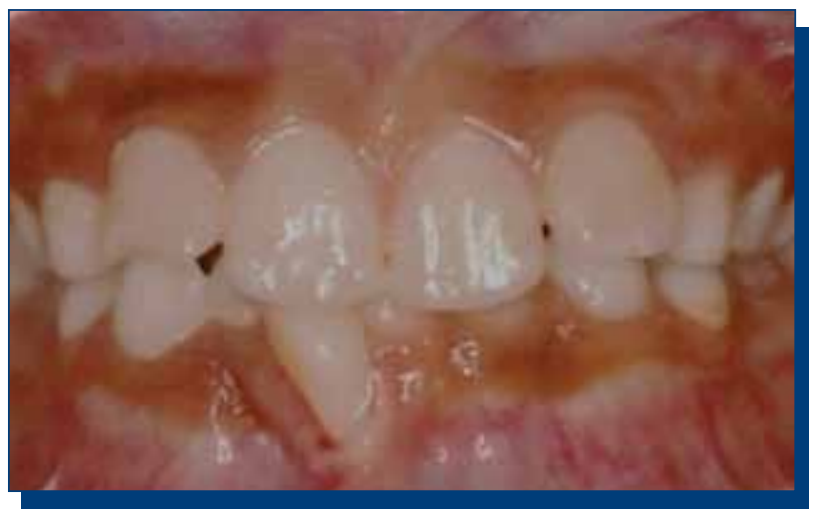

Figura 20. Vista anterior en donde se observa la marcada sobremordida vertical y la recesión en el órgano dentario incisivo central inferior derecho. 

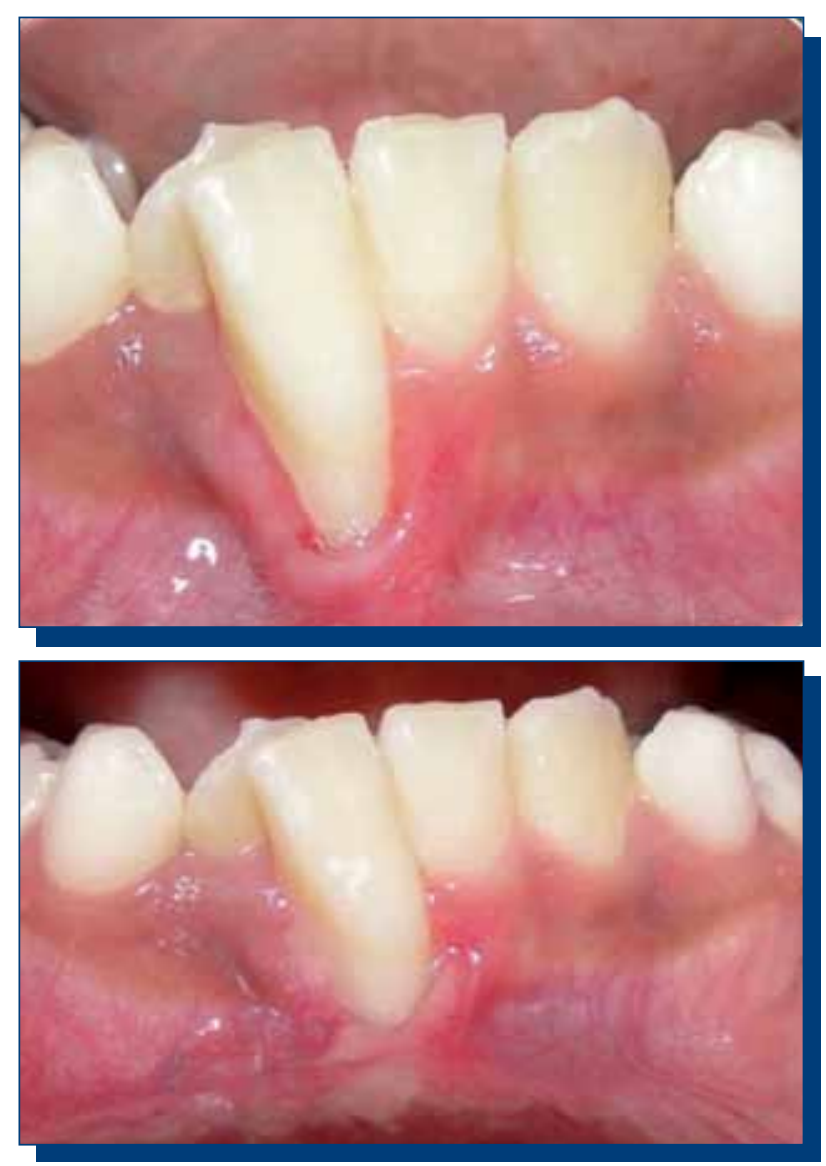

Figuras 21 y 22. Vista de la recesión gingival a los 15 días después de la toma de biopsia.

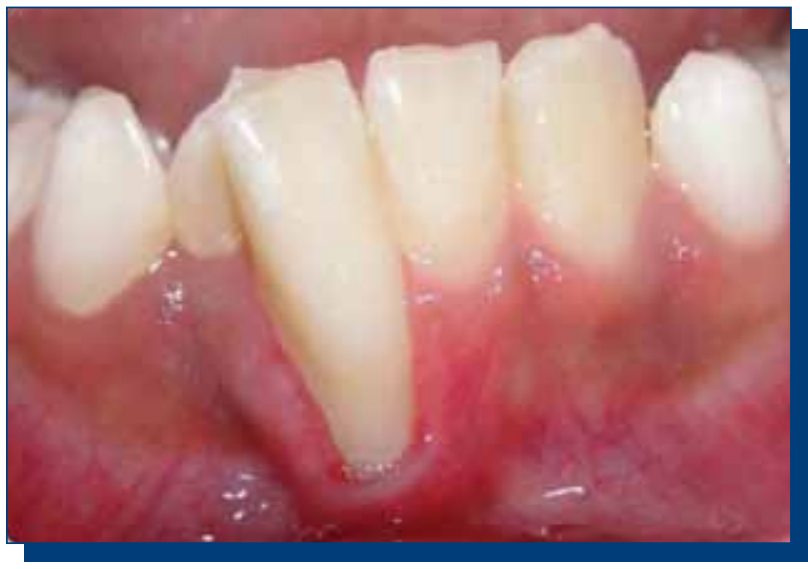

Figura 23. Vista anterior de la recesión en el incisivo central inferior derecho a los 3 meses.

\section{CONCLUSIONES}

La Hiperplasia Gingival Inflamatoria es una lesión poco común, multifactorial, con las que nos enfrentaremos en odontopediatría, de ahí la importancia de realizar un correcto diagnóstico multidisciplinario.

\section{REFERENCIAS BIBLIOGRÁFICAS}

1. Vargas PA, Pérez DEC, Jorge J, Rangel ALCA, Léon JE, Almeida OE. Denture hyperplasia with areas simulating oral inverted ductal papilloma. Med Oral Patol Oral Cir Bucal, 2005; 10 Suppl2: E117-121.

2. Espinosa ZM, Loza HG, Mondragón BR. Prevalencia de lesiones de la mucosa bucal en pacientes pediátricos: Informe preliminar. Cir Ciruj, 2006; 74(003): 153-157.

3. Carranza F, Takei H, Newman M. Periodontología Clínica. 9a edición. México, 2002: Editorial Mc Graw Hill.

4. Sapp JP, Eversole LR, Wysocki GP. Patología Oral y Maxilofacial Contemporánea. Editorial Harcourt-Mosby. España. Pp. 366-367.

5. Macedo LF, Dias JA, Antonio LG. Study of denture-induced fibrous hyperplasia cases diagnosed from 1979 to 2001. Quintessence Int, 2005; 36: 825-829.

6. Rodríguez AF, Sacsaquispe SJ. Hiperplasia Fibrosa Inflamatoria y posibles factores asociados en adultos mayores. Rev Estomatol Herediana, 2005; 15(2): 139-144.

7. Cawson R, Eveson J. Oral pathology \& diagnosis: Color atlas with integrated text. Editorial Saunders Company, USA.

8. Lindhe J, Karring T, Lang N. Clinical Periodontology \& Implant Dentistry. Editorial Blackwell-Munksgaard. $4^{\text {th }}$ edition. 2003, Slovenia.

9. Bascones Martínez A. Atlas de Patología de la Mucosa Bucal. Ediciones Avances. Madrid, 1989.
10. Palacios-Sánchez B, Cerero-Lapiedra R, Campo-Trapero J, Esparza Gómez GC. Alteraciones gingivales no relacionadas con placa. RCOE, 2006; 11(1): 43-55.

11. Gorlin R, Goldman H. Thoma: Patología Oral. Salvat editores. $2^{\mathrm{a}}$ reimpresión. 1981, España.

12. Arcos Castro M, Rojo Botello N, Quezada Rivera D. Estudio retrospectivo del año 2002 al 2006 prevalencia de granuloma piógeno, granuloma periférico de células gigantes y fibroma cemento-osificante periférico. Rev Odont Mex, 2008; 12(3): 137-141.

13. Regezi JA, Sciubba J. Patologia bucal. $2^{\mathrm{a}}$ ed. Editorial Interamericana, México, 1995

14. Strassburg M. Mucosa oral: Atlas a color de enfermedades. Libros Marban. $3^{a}$ edición. 1996, España.

15. McDonald R, Avery D, Dean J. Dentistry for the child and adolescent. Ed. Mosby. $8^{\text {th }}$ edition, 2004, USA.

16. Ramer M, Marrone J, Sthal B, Burakoff R. Hereditary gingival fibromatosis: Identification, treatment, control. J Am Dent Assoc, 1996; 127: 493-495.

17. Moawia M, Kassab y Cohen R. The etiology and prevalence of gingival recession. J Am Dent Assoc, 2003; 134: 220-225. 